\title{
Whole-exome sequencing identifies MYO15A mutations as a cause of autosomal recessive nonsyndromic hearing loss in Korean families
}

\author{
Hae-Mi Woo ${ }^{1+}$, Hong-Joon Park ${ }^{2 \dagger}$, Jeong-In Baek ${ }^{3 \dagger}$, Mi-Hyun Park', Un-Kyung Kim ${ }^{3}$, Borum Sagong ${ }^{3}$ \\ and Soo Kyung Koo ${ }^{1 *}$
}

\begin{abstract}
Background: The genetic heterogeneity of hearing loss makes genetic diagnosis expensive and time consuming using available methods. Whole-exome sequencing has recently been introduced as an alternative approach to identifying causative mutations in Mendelian disorders.

Methods: To identify the hidden mutations that cause autosomal recessive nonsyndromic hearing loss (ARNSHL), we performed whole-exome sequencing of 13 unrelated Korean small families with ARNSHL who were negative for GJB2 or SLC26A4 mutations.

Results: We found two novel compound heterozygous mutations, IVS11 + 1 and p.R2146Q, of MYO15A in one (SR903 family) of the 13 families with ARNSHL. In addition to these causative mutations, 13 nonsynonymous variants, including variants with uncertain pathogenicity (SR285 family), were identified in the coding exons of MYO15A from Korean exomes.

Conclusion: This is the first report of MYO15A mutations in an East Asian population. We suggest that close attention should be paid to this gene when performing genetic testing of patients with hearing loss in East Asia. The present results also indicate that whole-exome sequencing is a valuable method for comprehensive medical diagnosis of a genetically heterogeneous recessive disease, especially in small-sized families.
\end{abstract}

Keywords: Hearing loss, MYO15A, Mutation, Whole-exome sequencing

\section{Background}

Hearing loss is one of the most common sensory disorders and a heterogeneous trait in human beings [1]. More than $50 \%$ of prelingual deafness is estimated to have a genetic etiology, of which about $70 \%$ is nonsyndromic hearing loss (NSHL). Eighty percent of NSHL is autosomal recessive nonsyndromic hearing loss (ARNSHL). To date, more than 100 mapped loci have been reported, and 55 nonsyndromic hearing loss genes have been identified (http://hereditaryhearingloss.org/).

The genes that are most commonly implicated in ARNSHL are in order of frequency: GJB2, SLC26A4,

\footnotetext{
*Correspondence: skkoo@nih.go.kr

${ }^{\dagger}$ Equal contributors

'Division of Intractable Diseases, Center for Biomedical Sciences, National Institute of Health, Chungcheongbuk-do 363-951, South Korea Full list of author information is available at the end of the article
}

MYO15A, OTOF, and CDH23 [2]. Mutations in these genes do not occur at the same frequencies across ethnicities. Thus, the current genetic diagnosis of hearing loss is limited to common mutations in a patient's population of origin and relies on a gene-specific Sanger sequencing approach. This method is useful in some populations. For example, in Caucasians, mutation of a single gene, GJB2, accounts for up to $50 \%$ of all cases of ARNSHL [3]. However, in East Asian populations, GJB2 is responsible for a much lower percentage of deafness cases, and other genes associated with this disease remain largely unknown [4].

Mutations in $M Y O 15 \mathrm{~A}$ are among the most frequent causes of ARNSHL worldwide [2]. Forty-three mutations have been reported in MYO15A, most of which have been found by linkage analysis in consanguineous families from specific countries, such as Pakistan, Turkey,

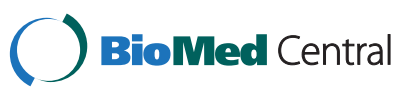


and Iran [5-7]. Diagnostic testing for this gene is not routinely offered owing to its large size, and the frequency and spectrum of MYO15A mutations in most ethnic populations are not known.

Recent advances in DNA enrichment and next-generation sequencing (NSG) technology have allowed rapid and cost-effective analysis of the causative mutations for human disorders [8]. Several studies have demonstrated the sensitivity and accuracy of the exome sequencing approach as well as the clinical utility of whole-exome sequencing $[9,10]$. In the present study, we applied whole-exome sequencing to 16 individuals from 13 unrelated families with ARNSHL. Of them, we report novel mutations and nonsynonymous MYO15A variants.

\section{Methods}

\section{Patients}

All affected individuals had bilateral and severe to profound hearing loss without additional symptoms, and had been diagnosed with hearing impairment at an early age. Informed consent was obtained from all participants, and this study was approved by the Institutional Review Board of the Korea National Institutes of Health (NIH). Genomic DNA was extracted from peripheral blood samples using a FlexiGene DNA extraction kit (QIAGEN, Hilden, Germany). Probands from each family were found to be negative for GJB2 and SLC26A4 mutations based on Sanger sequencing.

\section{Whole-exome sequencing}

Five micrograms of DNA were captured on the SeqCap EZ Human Exome Library v2.0 (Roche/NimbleGen, Madison, WI), following the manufacturer's protocol. We targeted all well-annotated protein-coding regions as defined by the CCDS (September 2009). Captured libraries were sequenced on the Solexa GAIIx Genome Analyzer (Illumina, San Diego, CA) with 78-bp paired-end reads, following the manufacturer's protocol. Reads were mapped to the reference human genome (GRCh37, UCSC hg19) using BWA (http://bio-bwa.sourceforge.net/). Single-nucleotide variants (SNVs) and insertions-deletions (indels) were identified using the SAMtools (http://samtools.sourceforge. net/), based on filtered variants with a mapping quality score $\geq 20$, and annotated using ANNOVAR (http://www. openbioinformatics.org/annovar/). Mutations identified by exome sequencing were confirmed by Sanger sequencing and genotyped with additional control individuals using TaqMan SNP Genotyping Assays (Applied Biosystems, Foster City, CA). Exome-based copy-number variation (CNV) was analyzed using the ExomeCNV programs [11].

\section{Sanger sequencing}

PCR and direct sequencing were used to prescreen GJB2 and SLC26A4 and to confirm the variants in the candidate genes that had been identified by exome sequencing. We also applied direct sequencing to read the missing regions (read depth $<5$ ) of candidate genes that were identified using Tablet - Next Generation Sequence Assembly Visualization [12]. The PCR products were directly sequenced, in both the forward and reverse directions, using an ABI 3730 instrument (Applied Biosystems). Each read was aligned to the reference sequence, and nucleotide changes were identified with the Sequencer software package (GeneCodes, Ann Arbor, MI).

\section{In silico analysis}

Evolutionary conservation of the sequences and structures of the proteins and nucleic acids was assessed using the ConSeq server (http://conseq.tau.ac.il/). The effect of the identified novel missense mutation was assessed using SIFT (http://sift.jcvi.org), PolyPhen-2 (http://genetics.bwh.harvard.edu/pph2/index.shtml), and MUpro (http://mupro.proteomics.ics.uci.edu/). These are automatic tools for predicting the impact of an amino acid substitution on the structure and function of a human protein. The 3D molecular structure of the MyTH4 domain was modeled using SWISS-MODEL Workplace (http://swissmodel.expasy.org/). The images were captured and rendered with the PYMOL software (http:// www.pymol.org/).

\section{Results}

\section{Whole-exome sequencing}

We performed whole-exome sequencing of 16 individuals from 13 unrelated families with ARNSHL, and found compound heterozygous mutations in the MYO15A gene from the SR-903 family, as well as a novel variant in the same gene from the SR-285 family (Additional file 1: Figure S1).

A summary of the exome sequencing results for the two families is provided in Table 1. On average, 97\% and $90 \%$ of the bases were covered to $1 \times$ and $10 \times$ within the targeted bases, respectively. The average number of observed variants was 55,828 . To identify pathogenic variants, we filtered out polymorphisms using the Single Nucleotide Polymorphism Database dbSNP131 and the Korean exome data, which includes exome data for 30 Koreans from another study [13], and the Korean genome database, TIARA [14]. We then focused on the variants of reported nonsyndromic deafness genes.

\section{Identification of causative mutations}

In samples SR-903 and SR-903B, the numbers of remaining variants were five and three, respectively (Table 1, Additional file 2: Table S1). Of these, three variants in MYO15A (IVS11 + 1, p.R2146Q, and p.S3474G) were shared by both siblings. The p.S3474G variant was 
Table 1 Results of whole-exome sequencing of three individuals with ARNSHL

\begin{tabular}{|c|c|c|c|}
\hline Parameter & SR-903 & SR-903B & SR-285 \\
\hline Total reads & $66,179,458$ & $74,672,946$ & $65,182,498$ \\
\hline Total yield (bp) & $5,161,997,724$ & $5,824,489,788$ & $5,084,234,844$ \\
\hline Mappable reads & $61,470,184$ & $68,485,482$ & $60,376,538$ \\
\hline Mappable yield (bp) & $4,669,705,365$ & $5,194,083,022$ & $4,596,079,334$ \\
\hline On-target reads & $39,914,806$ & $41,803,453$ & $36,505,698$ \\
\hline On-target yield (bp) & $2,458,150,580$ & $2,594,965,223$ & $2,203,746,888$ \\
\hline Coverage of target region (more than $10 x$ ) & $91 \%$ & $89 \%$ & $91 \%$ \\
\hline Mean read depth of targeted region & $56 \times$ & $59 x$ & $50 x$ \\
\hline Mean read depth of called variants & $46 \times$ & $50 x$ & $42 x$ \\
\hline Number of total variants & 56,505 & 54,340 & 56,640 \\
\hline Number of coding variants & 20,849 & 20,597 & 20,847 \\
\hline Number of missense, nonsense, splice, and indel variants & 10,633 & 10,425 & 10,574 \\
\hline After Korean control exome* filtering & 543 & 548 & 510 \\
\hline After dbSNP131 filtering & 457 & 472 & 447 \\
\hline Variants in reported deafness genes & 5 & 3 & 3 \\
\hline Shared variants & 3 & 3 & - \\
\hline
\end{tabular}

* Korean control exome dataset, which includes exome data for 30 Koreans from another study and the Korean genomes database, TIARA.

ruled out because it was reported in the 1000-genome database with a minor allele frequency (MAF) of 0.002, and it also appeared in dbSNP137(rs150181830). Sanger sequencing confirmed the other two variants in the affected siblings and their family. One variant, corresponding to p.R2140Q, was heterozygotic in the patient's father (SR-903 F). The other variant, IVS11 + 1G > A, was present in the mother (SR-903 M) (Figure 1).

SR-285 carried three candidate variants of known deafness genes (p.Q445R in GRHL2, p.T1321S in TECTA, and p.S2161F in MYO15A); only one mutation, p.S2161F in $M Y O 15 A$, was shared with an affected sibling (Figure 1). However, it was found to be in a heterozygous state without a second mutation. To search for other mutations, we subsequently sequenced the entire missing region (read depth <5) in all 66 exons of MYO15A (NM_016239). However, no additional mutation or small insertion or deletion was identified. In addition, no significantly suggestive copy-number variation $(\mathrm{CNV})$ region emerged from the exome-based CNV analysis. SR-285 was found to contain another MYO15A coding variant, p.G1220R, which was also shared by both affected sibling but was filtered out in the initial analysis due to its appearance in the Korean exome data (Additional file 2: Table $\mathrm{S} 2$ ). In the present study, we regard this variant as a polymorphism, although given the recessive nature of this gene, it is feasible that a low frequency in the general Korean population could render it pathogenic. Unfortunately, DNA samples were not available from the parents in this family and we could not confirm pathogenicity in a segregation study.

\section{In silico analysis of mutations detected in MYO15A}

Compound heterozygous mutations in MYO15A, IVS11 + 1 and p.R2146Q, were found in the SR-903 family, while a heterozygous variant, p.S2161F was identified in the SR-285 family. All of the probable causative mutations were novel and were not present in 409 ethnically matched controls (Table 2). The ConSeq server revealed that all of the causative mutations were located at a well-conserved site. Residues R2146 and S2161 are both located in the first MyTH4 domain of the MYO15A protein (Figure 2A). Figure 2B presents the locations of R2146 and S2161 in a 3D model of the MyTH4 domain based on PBD entry 3pvlA. In the first variant, the substitution of an uncharged, polar glutamine for arginine results in the loss of the positive charge on the arginine side-chain (p.R2146Q). In the other variant, the polar side chain of serine is replaced with a non-polar phenylalanine side-chain (p.S2161F). Both alterations are predicted by SIFT to be deleterious. The IVS11 + 1G > A alteration is predicted to affect splicing, thereby prematurely terminates.

\section{Identification of MYO15A polymorphism}

In addition to these causative mutations, 12 nonsynonymous variants were identified in the MYO15A coding exons from 17 hearing-loss patients and 30 Korean control 


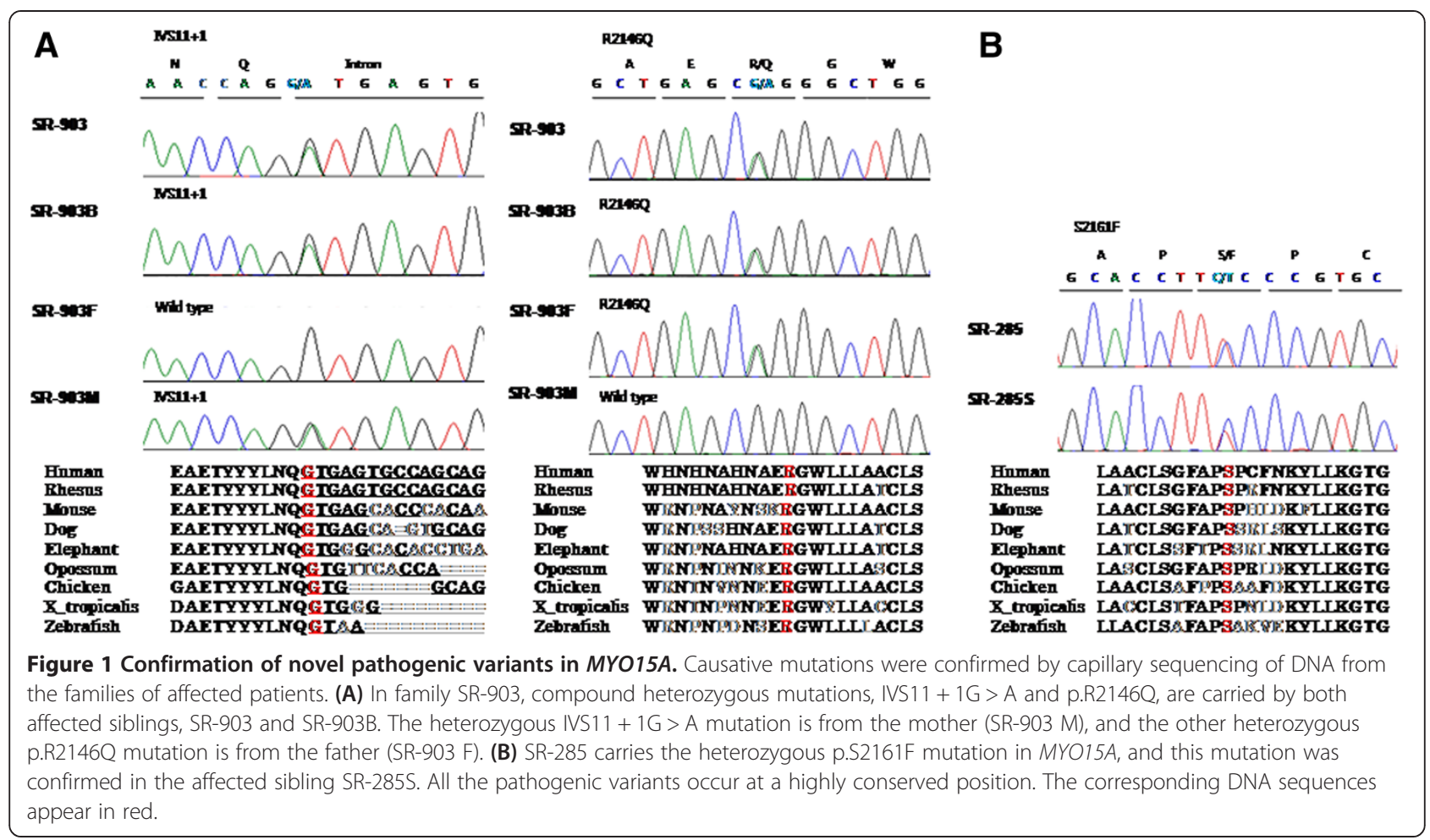

exomes (Additional file 2: Table S2). We consider these variants to be polymorphisms because they did not cosegregate with an affected member or were found in the controls.

\section{Discussion}

We performed whole-exome sequencing and identified novel MYO15A mutations in Korean families with ARNSHL. Comprehensive targeted enrichment and massive parallel sequencing have been employed for nonsyndromic hearing loss [15-17] and have focused only on the exons of all known deafness genes. However, the cost of whole-exome capture is not much more than that for only the deafness genes alone, and novel genes for hearing loss have been identified by a targeted NGS approach $[18,19]$. Thus, we targeted the whole exome to identify causative mutations in known deafness genes, as well as novel mutations responsible for hearing loss.

To identify pathogenic variants, we filtered out polymorphisms using the dbSNP131 and the Korean exome data. As an example, 1,462 variants in SR-903 remained after the dbSNP131 filtering. In contrast, after Korean exome filtering, 543 variants remained. This means that some variants that are commonly found in the Korean population are not present in dbSNP131. The application of Korean exome filtering more effectively removed

Table 2 Characteristics of the novel MYO15A mutations detected in this study

\begin{tabular}{|c|c|c|c|c|c|c|c|c|c|c|}
\hline \multirow[t]{2}{*}{ Family } & \multirow{2}{*}{$\begin{array}{c}\text { Genomic positions } \\
\text { (Hg19) }\end{array}$} & \multirow{2}{*}{$\begin{array}{l}\text { Nucleotide } \\
\text { change }\end{array}$} & \multirow{2}{*}{$\begin{array}{l}\text { Amino acid } \\
\text { change }\end{array}$} & \multirow[t]{2}{*}{ Location } & \multirow[t]{2}{*}{ Domain } & \multirow[t]{2}{*}{ Controls } & \multirow{2}{*}{$\begin{array}{l}\text { Evolutionary } \\
\text { conservation }\end{array}$} & \multicolumn{3}{|c|}{ In silico analysis } \\
\hline & & & & & & & & $\overline{\text { SIFT }^{1}}$ & ${\text { PolypPhen- } 2^{2}}^{2}$ & MUpro $^{3}$ \\
\hline \multicolumn{11}{|c|}{ Pathologic variant } \\
\hline SR-903 & Chr17:18035881 & $c .4320+1 G$ & IVS11 + 1 & Intron & Motor & $0 / 409$ & Yes & - & - & - \\
\hline SR-903 & Chr17:18049349 & C.G6437A & p.R2146Q & Exon 29 & MyTH4 & $0 / 409$ & Yes & 0 & 0.99 & -0.743 \\
\hline \multicolumn{11}{|c|}{ Variants with uncertain pathogenicity } \\
\hline SR-285 & Chr17:18049394 & c.C6482T & p.S2161F & Exon 29 & MyTH4 & 0/409 & Yes & 0 & 1 & -0.543 \\
\hline
\end{tabular}

${ }^{1}$ For SIFT, prediction of a change being deleterious $(<0.05)$ or tolerated.

${ }^{2}$ For PolyPhen-2, prediction of a change being deleterious $(>0.85)$, possibly deleterious $(0.15-0.85)$ or benign $(<0.15)$.

${ }^{3}$ For MUpro, prediction of a change that decreases protein stability (score $<0$ ) or increases protein stability (score $\left.>0\right)$, with a confidence score between -1 and 1 . The English in this document has been checked by at least two professional editors, both native speakers of English. For a certificate, please see: http://www.textcheck.com/certificate/GVv7E3. 


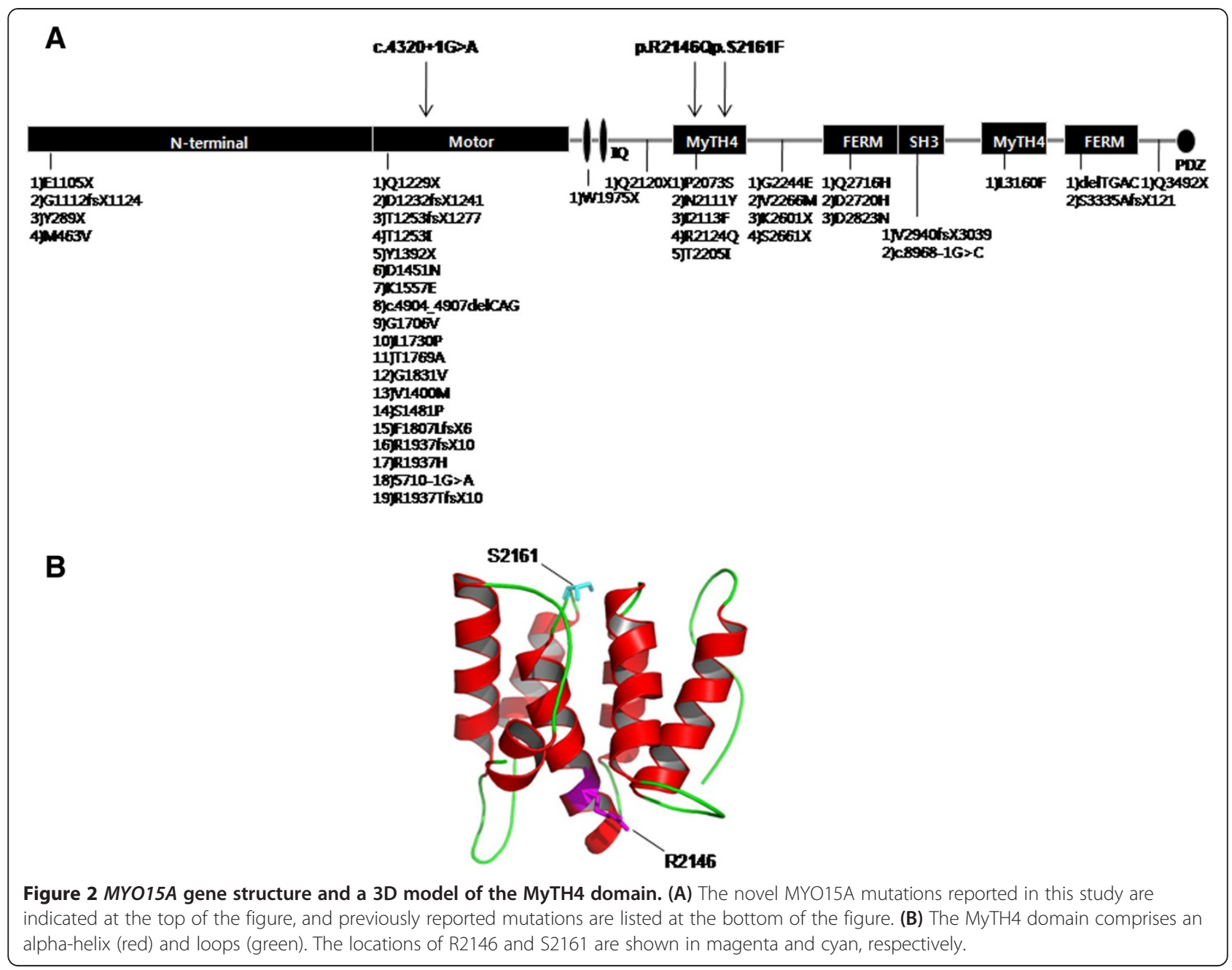

population-specific polymorphisms. A focus on known deafness genes also greatly reduced the number of candidate variants (Table 1). In sibling samples, the identification of causative mutations is straightforward, as the mutation must be present in both siblings.

MYO15A (66 coding exons) encodes an unconventional myosin (myosin XVA; 3,530 amino acids) that is expressed in the cochlea [20]. This protein has important roles in the differentiation and elongation of the inner ear hair cell stereocilia [21], and it is necessary for actin organization in hair cells [22]. Mutations that cause hearing loss were first identified at the DFNB3 locus, in residents of a village in Indonesia. Since then, many mutations have been reported from Pakistan, India, Turkey, Indonesia, and Brazil [5,7]. However, the two mutations identified in the present study are novel and are the first MYO15A mutations reported in East Asians.

IVS11 +1 is located in the motor domain of myosin XVA. The motor domain contains ATP- and actinbinding sites and produces the force that moves actin filaments in vitro [23]. Homozygous Shaker-2 (sh2) mice are deficient in myosin XVA due to a premature stop codon in the motor domain of the protein [24]. These mice exhibit profound deafness, similar to that observed in human DFNB3 patients. There are many reported mutations in this domain, and we propose that these mutations probably have deleterious effects on protein function.

Two missense mutations, p.S2161F and p.R2146Q, are located in exon 29, the first MyTH4 domain. The MyTH4 domain of myosin has been implicated in microtubule binding, as well as in actin binding at the plasma membrane [25]. There are data to suggest that the MyTH4/FERM domain in myosin VIIA is required for its localization to stereocilia tips [25-27], a process that is essential for the formation of the transmembrane actin microfilament assembly complex at the stereocilia tips. A recent study revealed that many deafness-causing mutations occur in this domain, and provided mechanistic explanations for known deafnesscausing mutations in MYO7A MyTH4-FERM using 
structure-based sequence analysis [27]. In the case of myosin XVA, it has been reported that p.R2124Q and p.P2073S mutations located in the MyTH4 domain interfere with the interaction between myosin XVA and whirlin so as to prevent the formation of a complex that appears to be required for normal hearing [28]. In the present study, we identified two novel mutations, p.S2161F and p.R2146Q, which are located in the MyTH4 domain. These mutations may result in pathogenesis and deafness and may have important roles in the functions of myosin XVA. The p.R2146Q protein contains a prominent exposed hydrophobic pocket with positively charged residues and has a structure that is similar to the reported R1190 of MYO7A MyTH4FERM. This region may directly bind to CEN2 at the negatively charged residues, and substitution with uncharged residues leads to an approximately 10-fold decrease in the binding affinity between MFS and CEN [27]. Therefore, we speculate that the p.R2146Q mutation cripples the inter-MyTH4-FERM interface that could lead to pathogenesis and deafness.

In SR-285, we identified two novel nonsynomous variants, p.G1220R and p.S2161F, in the MYO15A gene. Both of these variants were shared with the affected sibling. However, as the heterozygous variant p.G1220R was present in the Korean control exome data, we regard it as a polymorphism. Consequently, we could not identify a second deleterious mutation in this family. However, another variant, p.S2161F, was absent in 409 ethnically matched controls and was predicted to have a pathogenic effect on protein structure or function by in silico analysis; we would not rule out the possibility of a causative MYO15A gene mutation in this family. There are two possible explanations for the hearing loss observed in this family: (1) p.G1220R contributes to the phenotype of the SR-285 family and has a low frequency in the general Korean population; and (2) a pathogenic mutation exists in the MYO15A gene but in an exon that was not covered by our sequencing or it lies within intronic regulatory sequences. This problem highlights a limitation of exome-based analyses. Alternatively, a pathogenic mutation exists in another known gene uncovered by WES or the causative mutation is in an as-yet-unidentified hearing loss gene. Even though p.S2161F was not the causative mutation in this family, it has the potential to exert pathogenic effects on protein function in other families with hearing loss, as supported by the in silico analysis. Further functional assays to prove the pathogenicity of this variant would be useful to confirm the deleterious nature of this variant.

The GJB2 and SLC26A4 genes are frequently implicated in ARNSHL, and screening for these genes is relatively easy. Therefore, many studies have focused on only these two genes. However, a strategy for screening other hearing loss genes is difficult, and Sanger sequencing of candidate genes with multiple exons, such as $M Y O 15 A$, is time-consuming. To screen simultaneously for each of the known deafness genes and to discover hidden mutations, we initially performed WES on 16 individuals from 13 unrelated smaller families with ARNSHL who could not be analyzed using the current genetic approach owing to insufficient genetic information following the exclusion of mutations in the GJB2 and $S L C 26 A 4$ genes using Sanger sequencing. We identified a compound heterozygous mutation in MYO15A in one family and a novel variant in the same gene from another family. To our knowledge, this is the first report of $M Y O 15 A$ mutations in an East Asian population. It seems likely that there are additional ARNSHL-causing MYO15A mutations, as the gene is large and mutation analysis is rare, since complementary linkage analysis has not been performed in East Asian populations. The exact frequency of such mutations will need to be determined in a future larger cohort mutation analysis and close attention should be paid to this gene when performing genetic testing of patients with hearing loss in East Asia.

\section{Conclusions}

In summary, we identified mutations in the MYO15A gene in a East Asian population for the first time using whole-exome sequencing. The present results indicate that whole-exome sequencing is a valuable method for comprehensive medical diagnosis and provides a valuable alternative to analyzing all known causative genes by conventional sequencing in a genetically heterogeneous disease, especially in small-sized families. In the future, the rapid discovery of hearing loss-related genes by whole-exome sequencing is expected and will provide a better understanding of the condition.

\section{Additional files}

Additional file 1: Figure S1. Pedigrees of two families with autosomal recessive nonsyndromic hearing loss. (A) Family SR-903 carries a compound heterozygous mutation in MYO15A, which is shared by both siblings. (B) In family SR-285, only one mutation, p.S2161F in MYO15A, is shared with the affected sibling. Filled symbols in each pedigree represent affected individuals. The proband is indicated by an arrow. Asterisks indicate available samples. The three individuals whose exomes were sequenced are shown in red.

Additional file 2: Table S1. Candidate variants identified in this study. Table S2. Nonsynonymous mutations considered to be noncausative variations in 16 hearing-loss patients and 30 Korean exomes from another study.

\section{Abbreviations}

NSHL: Nonsyndromic hearing loss; ARNSHL: Autosomal recessive nonsyndromic hearing loss; NGS: Next generation sequencing. 


\section{Competing interests}

The authors do not have any conflicts of interest, financial or otherwise, to declare.

\section{Authors' contributions}

HMW, MHP, UKK and SKK designed the study; HMW, MHP and SKK analyzed the data and wrote the article; HJP collected the samples and examined the family information; JIB and SB performed the sequencing analyses. All authors read and approved the final manuscript.

\section{Acknowledgments}

This work was supported by Korea National Institute of Health intramural research grants 4800-4845-300-210 (no. 2011-N63005-00) and 4800-4845-302210 (no. 2012-N61001-00).

\section{Author details}

'Division of Intractable Diseases, Center for Biomedical Sciences, National Institute of Health, Chungcheongbuk-do 363-951, South Korea. ${ }^{2}$ Soree Ear Clinic, Seoul, South Korea. ${ }^{3}$ Department of Biology, College of Natural Sciences, Kyungpook National University, Deagu 702-701, South Korea.

Received: 8 January 2013 Accepted: 9 July 2013

Published: 17 July 2013

\section{References}

1. Smith RJ, Bale JF Jr, White KR: Sensorineural hearing loss in children. Lancet 2005, 365(9462):879-890.

2. Hilgert N, Smith RJ, Van Camp G: Forty-six genes causing nonsyndromic hearing impairment: which ones should be analyzed in DNA diagnostics? Mutat Res 2009, 681(2-3):189-196.

3. Kenneson A, Van Naarden Braun K, Boyle C: GJB2 (connexin 26) variants and nonsyndromic sensorineural hearing loss: a HuGE review. Genet Med 2002, 4(4):258-274.

4. Guo YF, Liu XW, Guan J, Han MK, Wang DY, Zhao YL, Rao SQ, Wang QJ: GJB2, SLC26A4 and mitochondrial DNA A1555G mutations in prelingual deafness in Northern Chinese subjects. Acta Otolaryngol 2008, 128(3):297-303.

5. Fattahi Z, Shearer AE, Babanejad M, Bazazzadegan N, Almadani SN, Nikzat $N$, Jalalvand K, Arzhangi S, Esteghamat F, Abtahi R, et al: Screening for MY015A gene mutations in autosomal recessive nonsyndromic, GJB2 negative Iranian deaf population. Am J Med Genet A 2011, 158A(8):1857-1864.

6. Bashir R, Fatima A, Naz S: Prioritized sequencing of the second exon of MYO15A reveals a new mutation segregating in a Pakistani family with moderate to severe hearing loss. Eur J Med Genet 2012, 55(2):99-102.

7. Cengiz FB, Duman D, Sirmaci A, Tokgoz-Yilmaz S, Erbek S, Ozturkmen-Akay $H$, Incesulu A, Edwards YJ, Ozdag H, Liu XZ, et al: Recurrent and private MY015A mutations are associated with deafness in the Turkish population. Genet Test Mol Biomarkers 2010, 14(4):543-550.

8. Ng SB, Buckingham KJ, Lee C, Bigham AW, Tabor HK, Dent KM, Huff CD, Shannon PT, Jabs EW, Nickerson DA, et al: Exome sequencing identifies the cause of a mendelian disorder. Nat Genet 2009, 42(1):30-35.

9. Choi M, Scholl UI, Ji W, Liu T, Tikhonova IR, Zumbo P, Nayir A, Bakkaloglu A, Ozen S, Sanjad S, et al: Genetic diagnosis by whole exome capture and massively parallel DNA sequencing. Proc Natl Acad Sci U S A 2009, 106 (45):19096-19101.

10. Ng SB, Turner EH, Robertson PD, Flygare SD, Bigham AW, Lee C, Shaffer T, Wong $M$, Bhattacharjee A, Eichler EE, et al: Targeted capture and massively parallel sequencing of 12 human exomes. Nature 2009, 461(7261):272-276.

11. Sathirapongsasuti JF, Lee H, Lee HAJ, Horst B, Brunner G, Cochran AJ, Binder S, Quackenbush JF, Nelson S: Exome sequencing-based copy-number variation and loss of heterozygosity deterction: ExomeCNV. Bioinformatics 2011, 27(19):2648-2654.

12. Milne I, Bayer M, Cardle L, Shaw P, Stephen G, Wright F, Marshall D: Tabletnext generation sequence assembly visualization. Bioinformatics 2010, 26(3):401-402

13. Choi BO, Koo SK, Park MH, Rhee H, Yang SJ, Choi KG, Jung SC, Kim HS, Hyun YS, Nakhro K, et al: Exome sequencing is an efficient tool for genetic screening of Charcot-Marie-Tooth Disease. Hum Mutat 2012, 33(11):1610-1615
14. Hong D, Park SS, Ju YS, Kim S, Shin JY, Yu SB, Lee WC, Lee S, Park H, Kim Jl, et al: TIARA: a database for accurate analysis of multiple personal genomes based on cross-technology. Nucleic Acids Res 2011 39(Database issue):D883-D888.

15. Diaz-Horta O, Duman D, Foster J 2nd, Sirmaci A, Gonzalez M, Mahdieh N, Fotouhi N, Bonyadi M, Cengiz FB, Menendez I, et al: Whole-exome sequencing efficiently detects rare mutations in autosomal recessive nonsyndromic hearing loss. PLoS One 2012, 7(11):e50628.

16. Shearer AE, DeLuca AP, Hildebrand MS, Taylor KR, Gurrola J 2nd, Scherer S, Scheetz TE, Smith RJ: Comprehensive genetic testing for hereditary hearing loss using massively parallel sequencing. Proc Natl Acad Sci U S A 2010, 107(49):21104-21109.

17. Brownstein Z, Friedman LM, Shahin H, Oron-Karni V, Kol N, Rayyan AA Parzefall T, Lev D, Shalev S, Frydman M, et al: Targeted genomic capture and massively parallel sequencing to identify genes for hereditary hearing loss in middle eastern families. Genome Biol 2011, 12(9):R89.

18. Pierce SB, Walsh T, Chisholm KM, Lee MK, Thornton AM, Fiumara A, Opitz $J M$, Levy-Lahad E, Klevit RE, King MC: Mutations in the DBP-deficiency protein HSD17B4 cause ovarian dysgenesis, hearing loss, and ataxia of Perrault Syndrome. Am J Hum Genet 2010, 87(2):282-288.

19. Walsh T, Shahin H, Elkan-Miller T, Lee MK, Thornton AM, Roeb W, Abu Rayyan A, Loulus S, Avraham KB, King MC, et al: Whole exome sequencing and homozygosity mapping identify mutation in the cell polarity protein GPSM2 as the cause of nonsyndromic hearing loss DFNB82. Am J Hum Genet 2010, 87(1):90-94.

20. Liang Y, Wang A, Belyantseva IA, Anderson DW, Probst FJ, Barber TD, Miller W, Touchman JW, Jin L, Sullivan SL, et al: Characterization of the human and mouse unconventional myosin XV genes responsible for hereditary deafness DFNB3 and shaker 2. Genomics 1999, 61(3):243-258.

21. Belyantseva IA, Boger ET, Friedman TB: Myosin XVa localizes to the tips of inner ear sensory cell stereocilia and is essential for staircase formation of the hair bundle. Proc Natl Acad Sci U S A 2003, 100(24):13958-13963.

22. Berg JS, Powell BC, Cheney RE: A millennial myosin census. Mol Biol Cell 2001, 12(4):780-794.

23. Redowicz MJ: Myosins and deafness. J Muscle Res Cell Motil 1999, 20 (3):241-248.

24. Probst FJ, Fridell RA, Raphael $Y$, Saunders TL, Wang A, Liang Y, Morell RJ Touchman JW, Lyons RH, Noben-Trauth K, et al: Correction of deafness in shaker- 2 mice by an unconventional myosin in a BAC transgene. Science 1998, 280(5368):1444-1447.

25. Weber KL, Sokac AM, Berg JS, Cheney RE, Bement WM: A microtubulebinding myosin required for nuclear anchoring and spindle assembly. Nature 2004, 431(7006):325-329.

26. Mburu P, Mustapha M, Varela A, Weil D, El-Amraoui A, Holme RH, Rump A, Hardisty RE, Blanchard S, Coimbra RS, et al: Defects in whirlin, a PDZ domain molecule involved in stereocilia elongation, cause deafness in the whirler mouse and families with DFNB31. Nat Genet 2003, 34(4):421-428

27. Wu L, Pan L, Wei Z, Zhang M: Structure of MyTH4-FERM domains in myosin VIla tail bound to cargo. Science 2011, 331(6018):757-760.

28. Shearer AE, Hildebrand MS, Webster JA, Kahrizi K, Meyer NC, Jalalvand K, Arzhanginy S, Kimberling WJ, Stephan D, Bahlo M, et al: Mutations in the first MyTH4 domain of MYO15A are a common cause of DFNB3 hearing loss. Laryngoscope 2009, 119(4):727-733.

doi:10.1186/1471-2350-14-72

Cite this article as: Woo et al:: Whole-exome sequencing identifies MYO15A mutations as a cause of autosomal recessive nonsyndromic hearing loss in Korean families. BMC Medical Genetics 2013 14:72. 